\title{
On Geodesics of Warped Sasaki Metric
}

\author{
Abderrahim Zagane* and Mustapha Djaa
}

(Communicated by Ákos G. HORVÁTH)

\begin{abstract}
In this paper we establish a necessary and sufficient conditions under which a curve be a geodesic respect to the warped Sasaki metric.
\end{abstract}

Keywords: Horizontal lift; vertical lift; warped Sasaki metric; geodesic.

AMS Subject Classification (2010): Primary: 53C22; 53C25 ; Secondary: 53A45; 53C20; 58E20.

*Corresponding author

\section{Introduction}

The main idea in this note consists in the modification of the Sasaki metric. First we introduce a new metric called warped Sasaki metric on the tangent bundle $T M$. This new natural metric will lead us to interesting results. Afterward we establish a necessary and sufficient conditions under which a curve be a geodesic with respect to the warped Sasaki metric.

\section{Basic Notions and Definition on $T M$.}

\section{Horizontal and vertical lifts on $T M$.}

Let $(M, g)$ be an m-dimensional Riemannian manifold and $(T M, \pi, M)$ be its tangent bundle. A local chart $\left(U, x^{i}\right)_{i=1 \ldots n}$ on $M$ induces a local chart $\left(\pi^{-1}(U), x^{i}, y^{i}\right)_{i=1 \ldots n}$ on $T M$. Denote by $\Gamma_{i j}^{k}$ the Christoffel symbols of $g$ and by $\nabla$ the Levi-Civita connection of $g$.

We have two complementary distributions on $T M$, the vertical distribution $\mathcal{V}$ and the horizontal distribution $\mathcal{H}$, defined by :

$$
\begin{aligned}
\mathcal{V}_{(x, u)} & =\operatorname{Ker}\left(d \pi_{(x, u)}\right)=\left\{\left.a^{i} \frac{\partial}{\partial y^{i}}\right|_{(x, u)} ; \quad a^{i} \in \mathbb{R}\right\} \\
\mathcal{H}_{(x, u)} & =\left\{\left.a^{i} \frac{\partial}{\partial x^{i}}\right|_{(x, u)}-\left.a^{i} u^{j} \Gamma_{i j}^{k} \frac{\partial}{\partial y^{k}}\right|_{(x, u)} ; \quad a^{i} \in \mathbb{R}\right\},
\end{aligned}
$$

where $(x, u) \in T M$, such that $T_{(x, u)} T M=\mathcal{H}_{(x, u)} \oplus \mathcal{V}_{(x, u)}$.

Let $X=X^{i} \frac{\partial}{\partial x^{i}}$ be a local vector field on $M$. The vertical and the horizontal lifts of $X$ are defined by

$$
\begin{aligned}
X^{V} & =X^{i} \frac{\partial}{\partial y^{i}} \\
X^{H} & =X^{i} \frac{\delta}{\delta x^{i}}=X^{i}\left\{\frac{\partial}{\partial x^{i}}-y^{j} \Gamma_{i j}^{k} \frac{\partial}{\partial y^{k}}\right\}
\end{aligned}
$$

Received : 07-May-2016, Accepted : 25-February-2017 

$T T M$.

For consequences, we have $\left(\frac{\partial}{\partial x^{i}}\right)^{H}=\frac{\delta}{\delta x^{i}}$ and $\left(\frac{\partial}{\partial x^{i}}\right)^{V}=\frac{\partial}{\partial y^{i}}$, then $\left(\frac{\delta}{\delta x^{i}}, \frac{\partial}{\partial y^{i}}\right)_{i=1 \ldots n}$ is a local adapted frame in Remark 2.1. .

1. if $w=w^{i} \frac{\partial}{\partial x^{i}}+\bar{w}^{j} \frac{\partial}{\partial y^{j}} \in T_{(x, u)} T M$, then its horizontal and vertical parts are defined by

$$
\begin{aligned}
w^{h} & =w^{i} \frac{\partial}{\partial x^{i}}-w^{i} u^{j} \Gamma_{i j}^{k} \frac{\partial}{\partial y^{k}} \in \mathcal{H}_{(x, u)} \\
w^{v} & =\left\{\bar{w}^{k}+w^{i} u^{j} \Gamma_{i j}^{k}\right\} \frac{\partial}{\partial y^{k}} \in \mathcal{V}_{(x, u)}
\end{aligned}
$$

2. if $u=u^{i} \frac{\partial}{\partial x^{i}} \in T_{x} M$ then its vertical and horizontal lifts are defined by

$$
\begin{aligned}
u^{V} & =u^{i} \frac{\partial}{\partial y^{i}} \in \mathcal{V}_{(x, u)} \in \mathcal{H}_{(x, u)} \\
u^{H} & =u^{i}\left\{\frac{\partial}{\partial x^{i}}-y^{j} \Gamma_{i j}^{k} \frac{\partial}{\partial y^{k}}\right\} .
\end{aligned}
$$

Proposition 2.1 ([16]). Let $(M, g)$ be a Riemannian manifold and $R$ its curvature tensor, then for all vector fields $X, Y \in$ $\Gamma(T M)$ and $p \in T^{2} M$ we have:

1. $\left[X^{H}, Y^{H}\right]_{p}=[X, Y]_{p}^{H}-\left(R_{x}(X, Y) u\right)^{V}$,

2. $\left[X^{H}, Y^{V}\right]_{p}=\left(\nabla_{X} Y\right)_{p}^{V}$,

3. $\left[X^{V}, Y^{V}\right]_{p}=0$,

where $p=(x, u)$.

\section{Warped Sasaki metric.}

\section{Warped Sasaki metric.}

Definition 3.1. Let $(M, g)$ be a Riemannian manifold and $f: M \times \mathbb{R} \rightarrow] 0,+\infty[$ be a smooth function. On the tangent bundle $T M$ we define a warped Saski metric noted $g_{f}^{S}$ by

1. $g_{f}^{S}\left(X^{H}, Y^{H}\right)_{(x, u)}=g_{x}(X, Y)$

2. $g_{f}^{S}\left(X^{H}, Y^{V}\right)_{(x, u)}=g_{f}^{S}\left(X^{V}, Y^{H}\right)_{(x, u)}=0$

3. $g_{f}^{S}\left(X^{V}, Y^{V}\right)_{(x, u)}=f(x, r) g_{x}(X, Y)$

where $X, Y \in \Gamma(T M),(x, u) \in T M$ and $r=g(u, u) . f$ is called warping function.

Note that, if $f=1$ then $g_{f}^{S}$ is the Sasaki metric [16].

The notion of Sasaki metric and Gromol-Chegeer metric was considered in [1], [12], [13], [14], [15], [16].

Lemma 3.1. Let $(M, g)$ be a Riemannian manifold, then for all $x \in M$ and $u=u^{i} \frac{\partial}{\partial x^{i}} \in T_{x} M$, we have the following

1. $X^{H}(g(u, u))_{(x, u)}=0$

2. $X^{H}(g(Y, u))_{(x, u)}=g\left(\nabla_{X} Y, u\right)_{x}$ 
3. $X^{V}\left(g(u, u)_{(x, u)}=2 g(X, u)_{x}\right.$

4. $X^{V}\left(g(Y, u)_{(x, u)}=g(X, Y)_{x}\right.$

Proof. Localy, if $U: x \in M \rightarrow U_{x}=u^{i} \frac{\partial}{\partial x^{i}} \in T M$ be a local vector field constant on each fiber $T_{x} M$, then from formulas (2.1) and (2.2) we obtain :

$$
\begin{aligned}
\text { 1. } X^{H}(g(u, u))_{(x, u)} & =\left[X^{i} \frac{\partial}{\partial x^{i}} g_{s t} y^{s} y^{t}-\Gamma_{i j}^{k} X^{i} y^{j} \frac{\partial}{\partial y^{k}} g_{s t} y^{s} y^{t}\right]_{(x, u)} \\
& =X\left(g(U, U)_{x}-2\left(\Gamma_{i j}^{k} X^{i} y^{j} g_{s k} y^{s}\right)_{(x, u)}\right. \\
& =\left(X\left(g(U, U)_{x}-2 g\left(U, \nabla_{X} U\right)\right)_{x}\right. \\
& =0 . \\
\text { 2. } X^{H}(g(Y, u))_{(x, u)} & =\left[X^{i} \frac{\partial}{\partial x^{i}} g_{s t} Y^{s} y^{t}-\Gamma_{i j}^{k} X^{i} y^{j} \frac{\partial}{\partial y^{k}} g_{s t} Y^{s} y^{t}\right]_{(x, u)} \\
& =X\left(g(Y, U)_{x}-\left(\Gamma_{i j}^{k} X^{i} y^{j} g_{s k} Y^{s}\right)_{(x, u)}\right. \\
& =\left(X\left(g(Y, U)_{x}-g\left(Y, \nabla_{X} U\right)\right)_{x}\right. \\
& \left.=g\left(\nabla_{X} Y, U\right)\right)_{x} . \\
\text { 3. } \quad X^{V}(g(u, u))_{(x, u)} & =\left[X^{i} \frac{\partial}{\partial y^{i}} g_{s t} y^{s} y^{t}\right]_{(x, u)}=2 X^{i} g_{i t} u^{t}=2 g(X, u)_{x} \\
4 . \quad X^{V}(g(Y, u))_{(x, u)} & =\left[X^{i} \frac{\partial}{\partial y^{i}} g_{s t} Y^{s} y^{t}\right]_{(x, u)}=X^{i} g_{s i} Y^{s}=g(X, Y)_{x}
\end{aligned}
$$

From Lemma 3.1, we obtain

Lemma 3.2. Let $(M, g)$ be a Riemannian manifold, $\left.F:(s, t) \in \mathbb{R}^{2} \rightarrow F(s, t) \in\right] 0,+\infty[, \alpha: M \rightarrow] 0,+\infty[$ and $\beta: \mathbb{R} \rightarrow$ ] $0,+\infty[$ be smooth functions. If $f(x, r)=F(\alpha(x), \beta(r))$, then we have the following

1. $X^{V}(f)_{(x, u)}=2 \beta^{\prime}(r) g_{x}(X, u) \frac{\partial F}{\partial t}(\alpha(x), \beta(r))$

2. $X^{H}(f)_{(x, u)}=g_{x}\left(\operatorname{grad}_{M} \alpha, X\right) \frac{\partial F}{\partial s}(\alpha(x), \beta(r))$

where $(x, u) \in T M$ and $r=g_{x}(u, u)$.

In the following, we consider $f(x, r)=F(\alpha(x), \beta(r))$, where $\left.F:(s, t) \in \mathbb{R}^{2} \rightarrow F(s, t) \in\right] 0,+\infty[, \alpha: M \rightarrow] 0,+\infty[$ and $\beta: \mathbb{R} \rightarrow] 0,+\infty[$ are smooth functions.

Theorem 3.1. Let $(M, g)$ be a Riemannian manifold. If $f(x, r)=F(\alpha(x), \beta(r))$ and $\nabla$ (resp $\widetilde{\nabla})$ denote the Levi-Civita connection of $(M, g)\left(\operatorname{resp}\left(T M, g_{f}^{S}\right)\right.$, then we have:

1. $\left(\widetilde{\nabla}_{X^{H}} Y^{H}\right)_{p}=\left(\nabla_{X} Y\right)_{p}^{H}-\frac{1}{2}\left(R_{x}(X, Y) u\right)^{V}$,

2. $\left(\widetilde{\nabla}_{X^{H}} Y^{V}\right)_{p}=\left(\nabla_{X} Y\right)_{p}^{V}+\frac{f(x, r)}{2}\left(R_{x}(u, Y) X\right)^{H}$

$$
+\frac{1}{2 f(x, r)} g_{x}\left(\operatorname{grad}_{M} \alpha, X\right) \frac{\partial F}{\partial s}(\alpha(x), \beta(r)) Y_{p}^{V}
$$

3. $\left.\left(\widetilde{\nabla}_{X^{V}} Y^{H}\right)_{p}=\frac{f(x, r)}{2}\left(R_{x}(u, X) Y\right)\right)^{H}+\frac{1}{2 f(x, r)} g_{x}\left(\operatorname{grad}_{M} \alpha, Y\right) \frac{\partial F}{\partial s}(\alpha(x), \beta(r)) X_{p}^{V}$

4. $\left.\left.\left(\widetilde{\nabla}_{X^{V}} Y^{V}\right)_{p}=\frac{\beta^{\prime}(r)}{f(x, r)} \frac{\partial F}{\partial t}(\alpha(x), \beta(r))\left[g_{x}(Y, U) X_{p}^{V}\right)+g_{x}(X, U) Y_{p}^{V}\right)-g_{x}(X, Y) U_{p}^{V}\right]$

$$
-\frac{1}{2} g_{x}(X, Y) \frac{\partial F}{\partial t}(\alpha(x), \beta(r))\left(\operatorname{grad}_{M} \alpha\right)_{p}^{H} .
$$

for all vector fields $X, Y \in \Gamma(T M)$ and $p=(x, u) \in T M$, where $R$ denote the curvature tensor of $(M, g)$. 
The proof of Theorem 3.1 follows directly from Kozul formula, Lemma 3.1 and Lemma 3.2.

Lemma 3.3. Let $(M, g)$ be a Riemannian manifold. If $X, Y \in \Gamma(T M)$ are vector fields and $(x, u) \in T M$ such that $X_{x}=u$, then we have

$$
d_{x} X\left(Y_{x}\right)=Y_{(x, u)}^{H}+\left(\nabla_{Y} X\right)_{(x, u)}^{V} .
$$

Proof. Let $\left(U, x^{i}\right)$ be a local chart on $M$ in $x \in M$ and $\left(\pi^{-1}(U), x^{i}, y^{j}\right)$ be the induced chart on $T M$, if $X_{x}=$ $\left.X^{i}(x) \frac{\partial}{\partial x^{i}}\right|_{x}$ and $Y_{x}=\left.Y^{i}(x) \frac{\partial}{\partial x^{i}}\right|_{x}$, then

$$
d_{x} X\left(Y_{x}\right)=\left.Y^{i}(x) \frac{\partial}{\partial x^{i}}\right|_{\left(x, X_{x}\right)}+\left.Y^{i}(x) \frac{\partial X^{k}}{\partial x^{i}}(x) \frac{\partial}{\partial y^{k}}\right|_{\left(x, X_{x}\right)},
$$

thus the horizontal part is given by

$$
\begin{aligned}
\left(d_{x} X\left(Y_{x}\right)\right)^{h} & =\left.Y^{i}(x) \frac{\partial}{\partial x^{i}}\right|_{\left(x, X_{x}\right)}-\left.Y^{i}(x) X^{j}(x) \Gamma_{i j}^{k}(x) \frac{\partial}{\partial y^{k}}\right|_{\left(x, X_{x}\right)} \\
& =Y_{\left(x, X_{x}\right)}^{H}
\end{aligned}
$$

and the vertical part is given by

$$
\begin{aligned}
\left(d_{x} X\left(Y_{x}\right)\right)^{v} & =\left.\left\{Y^{i}(x) \frac{\partial X^{k}}{\partial x^{i}}(x)+Y^{i}(x) X^{j}(x) \Gamma_{i j}^{k}(x)\right\} \frac{\partial}{\partial y^{k}}\right|_{\left(x, X_{x}\right)} \\
& =\left(\nabla_{Y} X\right)_{\left(x, X_{x}\right)}^{V} .
\end{aligned}
$$

\section{Geodesics of warped Sasaki metric}

\section{Lemma 4.1.}

Let $(M, g)$ be a Riemannian manifold and $x: I \rightarrow M$ be a curve on $M$. If $C: t \in I \rightarrow C(\tau)=(x(t), y(t)) \in T M$ is a curve in $T M$ such $y(t) \in T_{x(t)} M$ (i.e. $y(t)$ is a vector field along $x(t)$ ), then

$$
\dot{C}=\dot{x}^{H}+\left(\nabla_{\dot{x}} y\right)^{V}
$$

Proof. Locally, If $Y \in \Gamma(T M)$ is a vector field such $Y(x(t))=y(t)$ then we have

$$
\dot{C}(t)=d C(t)=d Y(x(t))
$$

Using Lemma 3.3 we obtain

$$
\dot{C}(t)=d Y(x(t))=\dot{x}^{H}+\left(\nabla_{\dot{x}} y\right)^{V}
$$

\section{Theorem 4.1.}

Let $(M, g)$ be a Riemannian manifold and $\left(T M, g_{f}^{S}\right)$ its tangent bundle equipped with the warped Sasaki metric. If $f(x, r)=F(\alpha(x), \beta(r))$ and $C(t)=(x(t), y(t))$ is curve on $T M$ such $y(t)$ is a vector field along $x(t))$, then

$$
\begin{aligned}
\widetilde{\nabla}_{\dot{C}} \dot{C} & =\left[\nabla_{\dot{x}} \dot{x}+f R\left(y, \nabla_{\dot{x}} y\right) \dot{x}-\frac{1}{2}\left\|\nabla_{\dot{x}} y\right\|^{2} \frac{\partial F}{\partial s} \operatorname{grad}_{M} \alpha\right]^{H} \\
& +\left[\nabla_{\dot{x}} \nabla_{\dot{x}} y+\left[\dot{x}(\alpha) \frac{\partial \ln F}{\partial s}+2 \beta^{\prime} \frac{\partial \ln F}{\partial t} g\left(\nabla_{\dot{x}} y, y\right)\right] \nabla_{\dot{x}} y-\beta^{\prime} \frac{\partial \ln F}{\partial t}\left\|\nabla_{\dot{x}} y\right\|^{2} y\right]^{V}
\end{aligned}
$$

Proof. 
We have

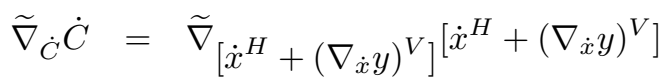

$$
\begin{aligned}
& =\widetilde{\nabla}_{\dot{x}^{H}} \dot{x}^{H}+\widetilde{\nabla}_{\dot{x}^{H}}\left(\nabla_{\dot{x}} y\right)^{V}+\widetilde{\nabla}_{\left(\nabla_{\dot{x}} y\right)^{V} \dot{x}^{H}+\widetilde{\nabla}_{\left(\nabla_{\dot{x}} y\right)^{V}}\left(\nabla_{\dot{x}} y\right)^{V}} \\
& =\left(\nabla_{\dot{x}} \dot{x}\right)^{H}-\frac{1}{2}(R(\dot{x}, \dot{x}) y)^{V}+\left(\nabla_{\dot{x}} \nabla_{\dot{x}} y\right)^{V}+\frac{f}{2}\left(R\left(y, \nabla_{\dot{x}} y\right) \dot{x}\right)^{H}+\frac{1}{2} \dot{x}(\alpha) \frac{\partial \ln F}{\partial s}\left(\nabla_{\dot{x}} y\right)^{V} \\
& +\frac{f}{2}\left(R\left(y, \nabla_{\dot{x}} y\right) \dot{x}\right)^{H}+\frac{1}{2} \dot{x}(\alpha) \frac{\partial \ln F}{\partial s}\left(\nabla_{\dot{x}} y\right)^{V} \\
& +\beta^{\prime} \frac{\partial \ln F}{\partial t}\left[g\left(\nabla_{\dot{x}} y, y\right)\left(\nabla_{\dot{x}} y\right)^{V}+g\left(\nabla_{\dot{x}} y, y\right)\left(\nabla_{\dot{x}} y\right)^{V}-g\left(\nabla_{\dot{x}} y, \nabla_{\dot{x}} y\right) y^{V}\right] \\
& -\frac{1}{2} g\left(\nabla_{\dot{x}} y, \nabla_{\dot{x}} y\right) \frac{\partial F}{\partial s}\left(\operatorname{grad}_{M} \alpha\right)^{H} \\
& \widetilde{\nabla}_{\dot{C}} \dot{C}=\left[\nabla_{\dot{x}} \dot{x}+f R\left(y, \nabla_{\dot{x}} y\right) \dot{x}-\frac{1}{2}\left\|\nabla_{\dot{x}} y\right\|^{2} \frac{\partial F}{\partial s} \operatorname{grad}_{M} \alpha\right]^{H} \\
& +\left[\nabla_{\dot{x}} \nabla_{\dot{x}} y+\dot{x}(\alpha) \frac{\partial \ln F}{\partial s} \nabla_{\dot{x}} y+\beta^{\prime} \frac{\partial \ln F}{\partial t}\left[2 g\left(\nabla_{\dot{x}} y, y\right) \nabla_{\dot{x}} y-\left\|\nabla_{\dot{x}} y\right\|^{2} y\right]\right]^{V} \\
& =\left[\nabla_{\dot{x}} \dot{x}+f R\left(y, \nabla_{\dot{x}} y\right) \dot{x}-\frac{1}{2}\left\|\nabla_{\dot{x}} y\right\|^{2} \frac{\partial F}{\partial s} \operatorname{grad}_{M} \alpha\right]^{H} \\
& +\left[\nabla_{\dot{x}} \nabla_{\dot{x}} y+\left[\dot{x}(\alpha) \frac{\partial \ln F}{\partial s}+2 \beta^{\prime} \frac{\partial \ln F}{\partial t} g\left(\nabla_{\dot{x}} y, y\right)\right] \nabla_{\dot{x}} y-\beta^{\prime} \frac{\partial \ln F}{\partial t}\left\|\nabla_{\dot{x}} y\right\|^{2} y\right]^{V}
\end{aligned}
$$

From the Theorem 4.1 we obtain

\section{Theorem 4.2.}

Let $(M, g)$ be a Riemannian manifold and $\left(T M, g_{f}^{S}\right)$ its tangent bundle equipped with the warped Sasaki metric. If $f(x, r)=F(\alpha(x), \beta(r))$ and $C(t)=(x(t), y(t))$ is curve on $T M$ such $y(t)$ is a vector field along $x(t))$, then $C$ is a geodesic on $T M$ if and only if

$$
\begin{cases}\nabla_{\dot{x}} \dot{x} & =\frac{1}{2}\left\|\nabla_{\dot{x}} y\right\|^{2} \frac{\partial F}{\partial s} \operatorname{grad}_{M} \alpha-f R\left(y, \nabla_{\dot{x}} y\right) \dot{x} \\ \nabla_{\dot{x}} \nabla_{\dot{x}} y & =\beta^{\prime} \frac{\partial \ln F}{\partial t}\left\|\nabla_{\dot{x}} y\right\|^{2} y-\left[\dot{x}(\alpha) \frac{\partial \ln F}{\partial s}+2 \beta^{\prime} \frac{\partial \ln F}{\partial t} g\left(\nabla_{\dot{x}} y, y\right)\right] \nabla_{\dot{x}} y\end{cases}
$$

Definition $4.1([16])$. Let $(M, g)$ be a Riemannian manifold and $\left(T M, g_{f}^{S}\right)$ its tangent bundle equipped with the warped Sasaki metric. A curve $C(t)=(x(t), y(t))$ is said to be a horizontal lift of the curve $x(t)$ if and only if $\nabla_{\dot{x}} y=0$.

Definition 4.2 ([16] [15]). Let $(M, g)$ be a Riemannian manifold and $\left(T M, g_{f}^{S}\right)$ its tangent bundle equipped with the warped Sasaki metric. If $x(t)$ is a curve on $(M, g)$, then the curve $C(t)=(x(t), \dot{x}(t))$ is called the natural lift of curve $x(t)$.

Using Theorem 4.2 we deduce:

Corollary 4.1. Let $(M, g)$ be a Riemannian manifold and $\left(T M, g_{f}^{S}\right)$ its tangent bundle equipped with the warped Sasaki metric. The natural lift $C(t)=(x(t), \dot{x}(t))$ of any geodesic $x(t)$ on $(M, g)$ is a geodesic on $\left(T M, g_{f}^{S}\right)$.

Corollary 4.2. Let $\left(M^{m}, g\right)$ be a Riemannian manifold, $\left(T M, g_{f}^{S}\right)$ its tangent bundle equipped with the warped Sasaki metric and $C(t)=(x(t), y(t))$ be a horizontal lift of the curve $x(t)$ (i.e. $\left.\nabla_{\dot{x}} y=0\right)$. Then $C(t)$ is a geodesic on $\left(T M, g_{f}^{S}\right)$ if and only if $\quad x(t)$ is a geodesic on $(M, g)$. 
Remark 4.1. If $C(t)=(x(t), y(t))$ is a horizontal lift of the curve $x(t)$ then locally we have

$$
\begin{aligned}
\nabla_{\dot{x}} y=0 & \Leftrightarrow \frac{d y^{s}}{d t}+\Gamma_{i j}^{s} y^{i} \frac{d x^{j}}{d t}=0 \\
& \Leftrightarrow y(t)=e^{-A(t)} K
\end{aligned}
$$

where $K \in \mathbb{R}^{m}$ and $A(t)=\left(\Gamma_{i j}^{s} \frac{d x^{j}}{d t}\right)_{s, i}$

Remark 4.2. Using the Remark 4.1 we can construct an infinity of examples of geodesics on $\left(T M, g_{f}^{S}\right)$.

Example 4.1. Condider the upper half-plane

$$
\left.\mathbb{R}_{+}^{2}=\left\{(x, y) \in \mathbb{R}^{2} \quad ; \quad y>0\right\}\right\}
$$

with the metric of Lobatchevski's non-euclidean geometry given by

$$
g_{11}=g_{22}=\frac{1}{y} \quad, \quad g_{12}=g_{21}=0 .
$$

The Christoffel symbols of the Riemannian connection are given by

$$
\Gamma_{11}^{1}=\Gamma_{22}^{1}=\Gamma_{12}^{2}=\Gamma_{21}^{2}=0, \quad \Gamma_{11}^{2}=\frac{1}{y}, \quad \Gamma_{22}^{2}=\Gamma_{12}^{1}=\Gamma_{21}^{1}=-\frac{1}{y} .
$$

1. If $C(t)=\left(x_{0}, y(t), u(t), v(t)\right)$ is horizontal lift of the curve $\left(x_{0}, y(t)\right)$, then the matrix $A(t)$ is given by

$$
A(t)=-\frac{1}{y}\left(\begin{array}{cc}
\frac{d y}{d t} & 0 \\
0 & \frac{d y}{d t}
\end{array}\right)
$$

and

$$
C(t)=\left(x_{0}, y(t), k_{1} y(t), k_{2} y(t)\right)
$$

2. If $C(t)=(x(t), y(t), u(t), v(t))$ is horizontal lift of the curve $(x(t), y(t))$ such $y(t)=a x(t)+b$ and $x \neq 0$, then the matrix $A(t)$ is given by

$$
A(t)=-\frac{d x}{(a x(t)+b) d t}\left(\begin{array}{cc}
a & -1 \\
1 & a
\end{array}\right)
$$

and

$$
C(t)=\left(x(t), y(t), \exp \left[\ln (y(t))\left(\begin{array}{cc}
a & -1 \\
1 & a
\end{array}\right)\right] K\right)
$$

where $K \in \mathbb{R}^{2}$

Example 4.2. Let $\mathbb{R}^{2}$ equipped with the Riemannian metric in polar coordinate defined by :

$$
g=d r^{2}+h(r, \theta)^{2} d \theta^{2}
$$

Relatively to the orthonormal frame

$$
e_{r}=\frac{\partial}{\partial r}, \quad e_{\theta}=\frac{1}{h(r, \theta)} \frac{\partial}{\partial \theta}
$$

we have

$$
\nabla_{e_{r}} e_{r}=\nabla_{e_{r}} e_{\theta}=0, \quad \nabla_{e_{\theta}} e_{r}=\frac{1}{h} \frac{\partial h}{\partial r} e_{\theta}, \quad \nabla_{e_{\theta}} e_{\theta}=-\frac{1}{h} \frac{\partial h}{\partial r} e_{r} .
$$

and the matrix of Levi-Civita connection relatively to the orthonormal frame $\left(e_{r}, e_{\theta}\right)$ is given by

$$
\Gamma=\left(\begin{array}{cc}
0 & -\frac{\partial h}{\partial r} d \theta \\
\frac{\partial h}{\partial r} d \theta & 0
\end{array}\right)
$$


If $C(t)=(r(t), \theta(t), u(t), v(t))$ is horizontal lift of the curve $(r(t), \theta(t))$ then matrix $A$ relatively to the orthonormal frame $\left(e_{r}, e_{\theta}\right)$ is given by

$$
A=\left(\begin{array}{cc}
0 & -\frac{1}{h} \frac{\partial h}{\partial r} \frac{d \theta}{d t} \\
\frac{1}{h} \frac{\partial h}{\partial r} \frac{d \theta}{d t} & 0
\end{array}\right)
$$

and

$$
\left\{\begin{array}{l}
u(t)=k_{1} \cos \left(\int \frac{1}{h} \frac{\partial h}{\partial r} \frac{d \theta}{d t}\right)+k_{2} \sin \left(\int \frac{1}{h} \frac{\partial h}{\partial r} \frac{d \theta}{d t}\right) \\
v(t)=-k_{1} \sin \left(\int \frac{1}{h} \frac{\partial h}{\partial r} \frac{d \theta}{d t}\right)+k_{2} \cos \left(\int \frac{1}{h} \frac{\partial h}{\partial r} \frac{d \theta}{d t}\right)
\end{array}\right.
$$

Theorem 4.3.

Let $(M, g)$ be a Riemannian manifold and $\left(T M, g_{f}^{S}\right)$ its tangent bundle equipped with the warped Sasaki metric and $x(t)$ be a geodesic on $M$. If $f(x, r)=F(\alpha(x), \beta(r))$ and $C=(x(t), y(t))$ is a geodesic on $T M$ such $\nabla_{\dot{x}} y \neq 0$ then

$$
\dot{x}(\alpha) \frac{\partial \ln F}{\partial s}(\alpha(x(t)), \beta(r(t)))=0
$$

where $r(t)=g_{x(t)}(y(t), y(t))$.

Proof.

Let $x(t)$ be a geodesic on $M$ then $\nabla_{\dot{x}} \dot{x}=0$. Using the first equation of formula (4.3) we obtain

$$
\begin{aligned}
g\left(\nabla_{\dot{x}} \dot{x}, \dot{x}\right)=0 & \Rightarrow \frac{1}{2}\left\|\nabla_{\dot{x}} y\right\|^{2} \frac{\partial F}{\partial s} g\left(\operatorname{grad}_{M} \alpha, \dot{x}\right)-f g\left(R\left(y, \nabla_{\dot{x}} y\right) \dot{x}, \dot{x}\right)=0 \\
& \Rightarrow \frac{1}{2}\left\|\nabla_{\dot{x}} y\right\|^{2} \dot{x}(\alpha) \frac{\partial F}{\partial s}(\alpha(x(t)), \beta(r(t)))=0 \\
& \Rightarrow \dot{x}(\alpha) \frac{\partial F}{\partial s}(\alpha(x(t)), \beta(r(t)))=0
\end{aligned}
$$

Corollary 4.3. Let $(M, g)$ be a Riemannian manifold and $\left(T M, g_{f}^{S}\right)$ its tangent bundle equipped with the warped Sasaki metric, $f(x, r)=\alpha(x)$ and $x(t)$ be a geodesic on $M$. If the curve $C=(x(t), y(t))$ is a geodesic on $T M$ such $\nabla_{\dot{x}} y \neq 0$, then $f$ is a constant along the curve $x(t)$.

The proof follows directly from Theorem 4.3.

\section{Corollary 4.4.}

Let $(M, g)$ be a Riemannian manifold and $\left(T M, g_{f}^{S}\right)$ its tangent bundle equipped with the warped Sasaki metric, $x(t)$ be a geodesic on $M$ and $f$ be a constant along the curve $x(t)$. If the curve $C=(x(t), y(t))$ is a geodesic on $T M$ such $\nabla_{\dot{x}} y \neq 0$ then $\nabla_{\dot{x}} \nabla_{\dot{x}} y=0$.

The proof follows directly from Theorem 4.3 and Theorem 4.2.

Corollary 4.5. Let $(M, g)$ be a Riemannian manifold, $\left(T M, g_{f}^{S}\right)$ its tangent bundle equipped with the warped Sasaki metric and $f(x, r)=f(x)=\alpha(x)$ be a constant along the curve $x(t)$. Then the curve $C=(x(t), y(t))$ is a geodesic on TM such $\nabla_{\dot{x}} y \neq 0$ if and only if we have

$$
\begin{cases}\nabla_{\dot{x}} \dot{x} & =f(x) R\left(\nabla_{\dot{x}} y, y\right) \dot{x} \\ \nabla_{\dot{x}} \nabla_{\dot{x}} y & =0 .\end{cases}
$$

Corollary 4.6. Let $(M, g)$ be a flat Riemannian manifold, $\left(T M, g_{f}^{S}\right)$ its tangent bundle equipped with the warped Sasaki metric and $f(x, r)=f(x)=\alpha(x)$ be a constant along the curve $x(t)$. Then the curve $C=(x(t), y(t))$ is a geodesic on TM such $\nabla_{\dot{x}} y \neq 0$ if and only if $x(t)$ is a geodesic on $M$ and

$$
\nabla_{\dot{x}} \nabla_{\dot{x}} y=0
$$




\section{Acknowledgement:}

The authors are supported by GMFAMI Laboratories and Algerian agency of research ANDRU.

\section{References}

[1] Cengiz, N., Salimov, A.A., Diagonal lift in the tensor bundle and its applications. Appl. Math. Comput. 142, no. 2-3, 309-319 (2003).

[2] Cherif, A.M., and Djaa, M., Geometry of energy and bienergy variations between Riemannian manifolds, Kyungpook Mathematical Journal, 55(2015), pp 715-730.

[3] Djaa M., Mohamed Cherif A., Zegga K. And Ouakkas S., On the Generalized of Harmonic and Bi-harmonic Maps, international electronic journal of geometry, 5 no. 1(2012), 90-100.

[4] Djaa M., Gancarzewicz J., The geometry of tangent bundles of order r, Boletin Academia , Galega de Ciencias ,Espagne, 4 (1985), 147-165

[5] Djaa, M., Djaa, N.E.H. and R. Nasri, Natural Metrics on T2M and Harmonicity, International Electronic Journal of Geometry Volume 6 No.1(2013), 100-111.

[6] Djaa N.E.H., Ouakkas S. , M. Djaa, Harmonic sections on the tangent bundle of order two. Annales Mathematicae et Informaticae 38( 2011) pp 15-25. 1.

[7] Djaa N.E.H., Boulal A. and Zagane A., Generalized warped product manifolds and Biharmonic maps, Acta Math. Univ. Comenianae; Vol. LXXXI, 2 (2012), 283-298.

[8] Djaa, N.E.H. and Djaa, M., Generalized Warped Product Manifold and Critical Riemannian Metric, Acta Mathematica Academiae Paedagogicae Nyiregyhaziensis Vol 28 (2012), 197-206.

[9] Elhendi, H., Terbeche, M. And Djaa, M., Tangent Bundle Of Order Two And Biharmonicity. Acta Math. Univ. Comenianae. Vol. 832 (2014). pp. 165-179.

[10] GEZER, A., On the tangent bundle with deformed Sasaki metric, Int. Electron. J. Geom. Volume 6 No. 2 (2013), 19-31.

[11] Gudmundsson, S. and Kappos, E.: On the Geometry of the Tangent Bundles, Expo. Math. 20, no.1(2002), 1-41.

[12] Salimov, A., Gezer, A., Akbulut, K., Geodesics of Sasakian metrics on tensor bundles. Mediterr. J. Math. 6, no.2, 135-147 (2009).

[13] Salimov, A., Gezer, A., On the geometry of the (1, 1)-tensor bundle with Sasaki type metric. Chinese Annals of Mathematics, Series B May 2011, Volume 32, Issue 3, pp 369-386.

[14] Salimov A. and Agca F. ,Some Properties of Sasakian Metrics in Cotangent Bundles. Mediterranean Journal of Mathematics; 8(2) (2011). 243-255.

[15] Salimov A. A. and Kazimova S., Geodesics of the Cheeger-Gromoll Metric, Turk J Math 33 (2009) , 99 - 105.

[16] Yano K., Ishihara S. Tangent and Cotangent Bundles, Marcel Dekker. INC. New York 1973.

\section{Affiliations}

\section{ABDERAHIM ZAGANE}

AdDress: Relizane University, Dept. of Mathematics, 48000, Relizane-Algeria.

E-MAIL: Zagane2014@outlook.fr

Mustapha DJAA

AdDress: Relizane University, Dept. of Mathematics, 48000, Relizane-Algeria.

E-MAIL: Djaamustapha@live.com 